\title{
PVA/LPRS Composite: Hot-pressing Preparation and Performance
}

\author{
Jian-mei Yin ${ }^{1, a}$ \\ ${ }^{1}$ Jiangxi Province Engineering Research Center of Ecological Chemical Industry, College of \\ Chemistry and Environmental Engineering, Jiujiang University, Qianjin Eath Road No.551, Jiujiang \\ City, Jiangxi Province, People's Republic of China \\ a624832940@qq.com
}

Keywords: Composite, Hot-pressing, Stretch, Strength.

\begin{abstract}
PVA/LPRS composite was prepared in order to deep explore manufacturing technology and performance-controlling methods of rice straw based composite. The preparation course includes the following procedures: rice straw drying, rice straw cutting, mixing with PVA and hot-pressing. The effects of hot-pressing time and temperature on performances of composite were studied. Results showed that tensile strength first increased and then decreased with hot-pressing time. The tensile strength arrived at 3.67 MPa when hot-pressing time was $12 \mathrm{~min}$, 51\% higher than that hot-pressed for $8 \mathrm{~min}$. Tensile elongation showed similar changing law as tensile strength. When hot-pressing time was $12 \mathrm{~min}$, the tensile elongation was 22.5\%. The hardness of composites was uneven and showed certain fluctuation. Hardness fluctuation range lay between 80 and 97 . Tensile strength first increased and then decreased with the increase of hot-pressing temperature. When hot-pressing temperature was $150{ }^{\circ} \mathrm{C}$, the tensile strength arrived at $3.41 \mathrm{MPa}, 85 \%$ higher than that of composite prepared at $130{ }^{\circ} \mathrm{C}$.
\end{abstract}

\section{Introduction}

Rice straw is one kind of important plant resource. It is also waste side-product of agriculture production. In modern times characterized by high standard of living, the application of rice straw becomes less and less and it is often burnt in the fields. Burning of rice straw has resulted in serious air pollution, which further harms health of people and interferes with transportation. If rice straw is utilized as recycling resource, related air pollution problem will be solved and furthermore resource and energy crisis can also be eased because rice straw can be converted to chemical raw materials and useful environmentally friendly composite. If such work is deep promoted, the consumption rate of non-renewable resources such as oil and natural gas will be decreased. Thankfully, studies converting rice straw to resource continuously reported. Ethanol is now one of the most widely used transport bio-fuels and production of ethanol from rice straw (RS) is an effective RS utilization way. Zhu et al investigated a novel two-step RS pretreatment process with the goal of decreasing the ethanol production cost through complete utilization of its components ${ }^{[1]}$. In the process, RS was first treated with dilute sulfuric acid to remove the hemicellulose and recover the xylose from its hydrolyzate as a feedstock for xylitol production, and then the residue was treated with the standard sulfomethylation reagent to remove the lignin and recover its hydrolyzate containing the lignosulfonate as a cement water reducer. After AT, the xylose was recovered from its hydrolyzate with $83.2 \%$ yield. After ST, its hydrolyzate containing 5.0\% lignosulfonate was directly recovered as a cement water reducer. Under the above best two-step treatment conditions, 94\% hemicellulose and 92\% lignin in the origin RS were removed, but cellulose had almost no loss. After the simultaneous saccharification and fermentation of the two-step treated RS $\left(100 \mathrm{~g} \mathrm{~L}^{-1}\right)$ for $72 \mathrm{~h}$, the ethanol concentration and its yield reached $40.6 \mathrm{~g} \mathrm{~L}^{-1}$ and $86.4 \%$ respectively. Liang et al studied the preparation and performance of novel lignocellulosic hybrid particleboard composites with low cost and high performance using the mixture of rice straws and coir fibers ${ }^{[2]}$. The coir fibers content had a significant negative linear effect on the bending properties and thickness swelling, but a significant positive linear effect on the internal bonding strength due to the lower wax and holocellulose content of coir fiber. Above 
mentioned reports are only typical examples utilizing rice straw as resource or extracting resource from rice straw, more related studies are easily found in literature ${ }^{[3-7]}$.

This work focuses on environmentally friendly composite using rice straw. Rice straw has good strength to support its fruit and so it is adapt to prepare composite. Different parts of rice straw have different biological and mechanical characteristics. This maybe affects performance of composite. Here lower part of rice straw (LPRS) based composite was prepared by hot-pressing method and its performance was studied.

\section{Materials and Methods}

Materials. Rice straw was obtained from Jiujiang suburbs farmland and was dried in the sun before use. Poly(vinyl alcohol) was purchased from Xiangwei company (Hunan, China) and was used as received. Water was from urban water-supplying system. Dimethicone oil was purchased from Xilong chemical company (Shantou, China) and was used as received.

Preparation of PVA/LPRS Composite. Rice straw was cut into two parts from the middle to collect LPRS. Then LPRS was cut into $1.5 \mathrm{~cm}$ segments. A series of $18 \mathrm{~g}$ LPRS segments were weighed into beaker, and then $10 \mathrm{~mL}$ water was added, followed by stirring for 5 min so that the LPRS was uniformly moistened. Thereafter, a series of $12 \mathrm{~g}$ PVA were weighed and added into moistened grass, followed by stirring for $5 \mathrm{~min}$. The mixed raw material was put in a $125 \mathrm{~mm} \times 125$ $\mathrm{mm} \times 2 \mathrm{~mm}$ iron mold and then was hot-pressed on MZ-3012 machine at 10MPa pressure. After cooling to room temperature, the product was taken out to be tested.

Measurement of Composite. $2.5 \mathrm{~cm}$ wide strips were cut out from products to test tensile performance. Tensile performances were measured on MZ-2000D.D1 electronic universal testing machine (Mingzhu test machine company, Jiangdu, Jiangsu, China) with tensile speed as $5 \mathrm{~mm} / \mathrm{min}$. Square with side length as $4 \mathrm{~cm}$ was selected from products and its four vertices were selected as hardness-measurement points. The four vertices were labeled respectively as point 1, 2, 3 and 4 according to hardness-increasing order. Shore hardness was measured with LX-A Shore A type durometer (Mingzhu test machine company, Jiangdu, Jiangsu, China).

\section{Results and Discussion}

The Effect of Hot-pressing Time on the Performance of Composite Materials. A series of PVA/LPRS composites were prepared at $140^{\circ} \mathrm{C}$ for different time. The change of tensile strengths with the increase of hot-pressing time was drawn in Fig. 1.

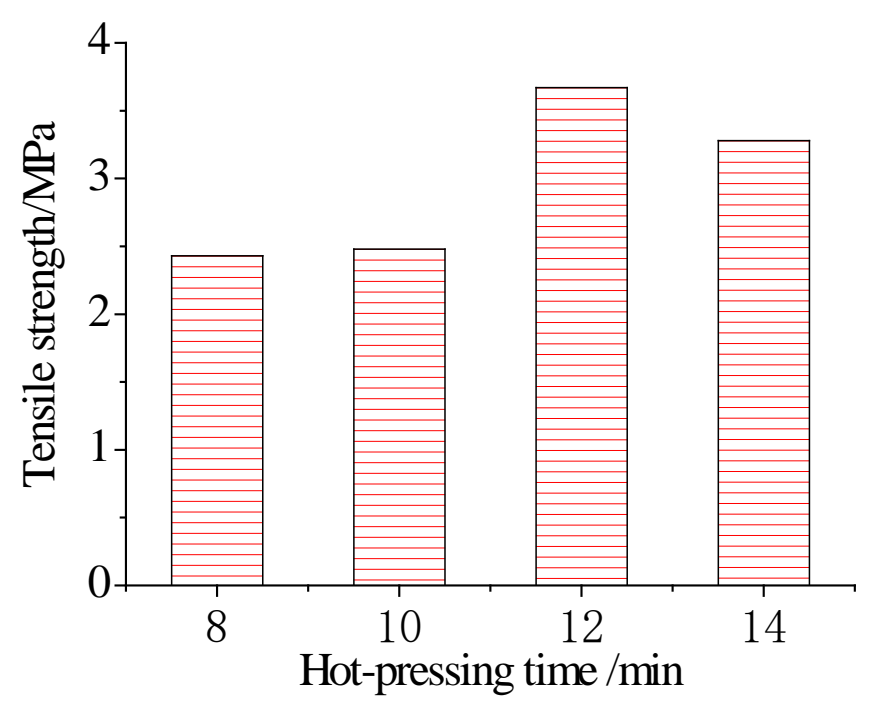

Fig. 1 The tensile strength of composites hot-pressed for different time. 
It can be seen that tensile strength first increases then decreases with hot-pressing time. Short hot-pressing time resulted in incomplete plasticization and distribution among LPRS. Long hot-pressing time led to structural change of LPRS and PVA. The tensile strength arrived at 3.67 MPa when hot-pressing time was $12 \mathrm{~min}, 51 \%$ higher than that hot-pressed for $8 \mathrm{~min}$.

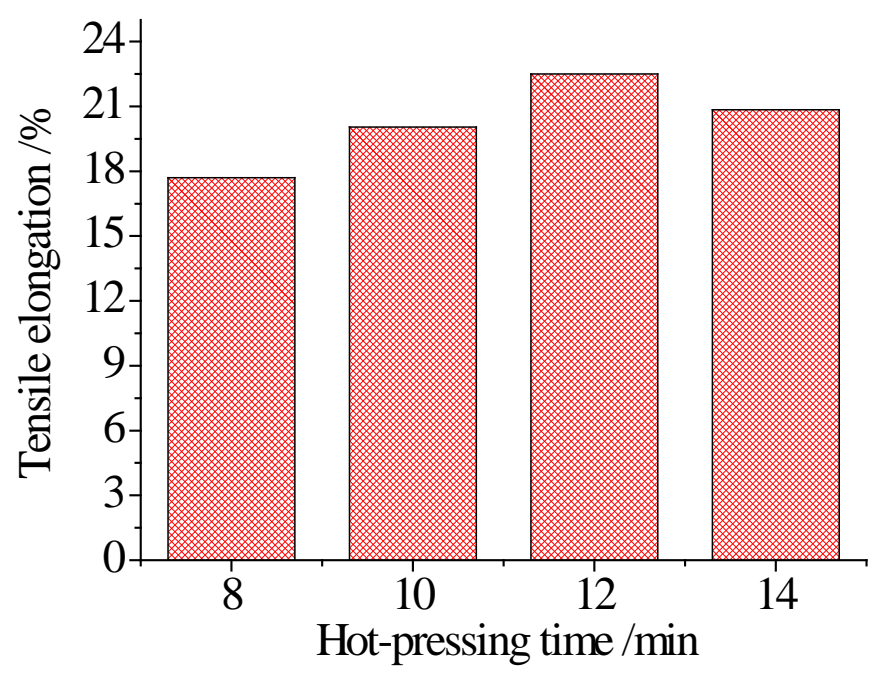

Fig. 2 The tensile elongation of composites hot-pressed for different time.

The change of tensile elongation with hot-pressing time was drawn respectively in Fig. 2. It can be seen that tensile elongation showed similar changing law as tensile strength. When hot-pressing time was $12 \mathrm{~min}$, the tensile elongation was 22.5\%. Composite hot-pressed for short time had lower strength and suffered shorter tensile course. Long hot-pressing time made tensile extending capability of composite decrease.

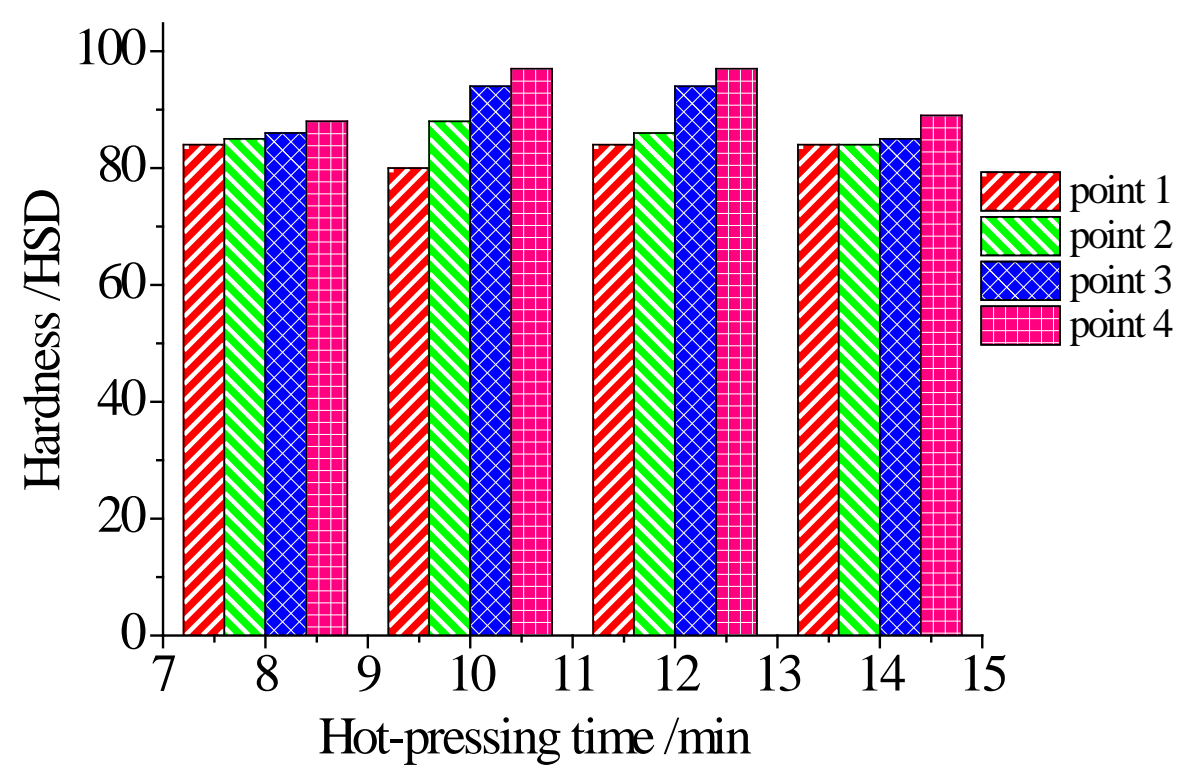

Fig. 3 The hardness of composites hot-pressed for different time.

The effect of hot-pressing time on the hardness of composites was shown in Fig. 3. It can be seen that the hardness of composites is uneven and showed certain fluctuation. Hardness fluctuation range lay between 80 and 97 . This perhaps resulted from non-uniform of surface composition. There existed tiny hole, LPRS part, PVA part and mixed part of LPRS and PVA. These surface morphologies exerted different effects to hardness management. 
The Effect of Hot-pressing Temperature on the Performance of Composite Materials. A series of PVA/LPRS composites were prepared at different hot-pressing temperatures. The change of tensile strengths with the increase of hot-pressing temperature was drawn in Fig. 4. It can be seen that tensile strength first increases then decreases with the increase of hot-pressing temperature. When hot-pressing temperature was $150{ }^{\circ} \mathrm{C}$, the tensile strength arrived at $3.41 \mathrm{MPa}, 85 \%$ higher than that of composite prepared at $130{ }^{\circ} \mathrm{C}$. Adapt hot-pressing temperature both promoted diffusion of PVA and prevented raw materials from structural disruption.

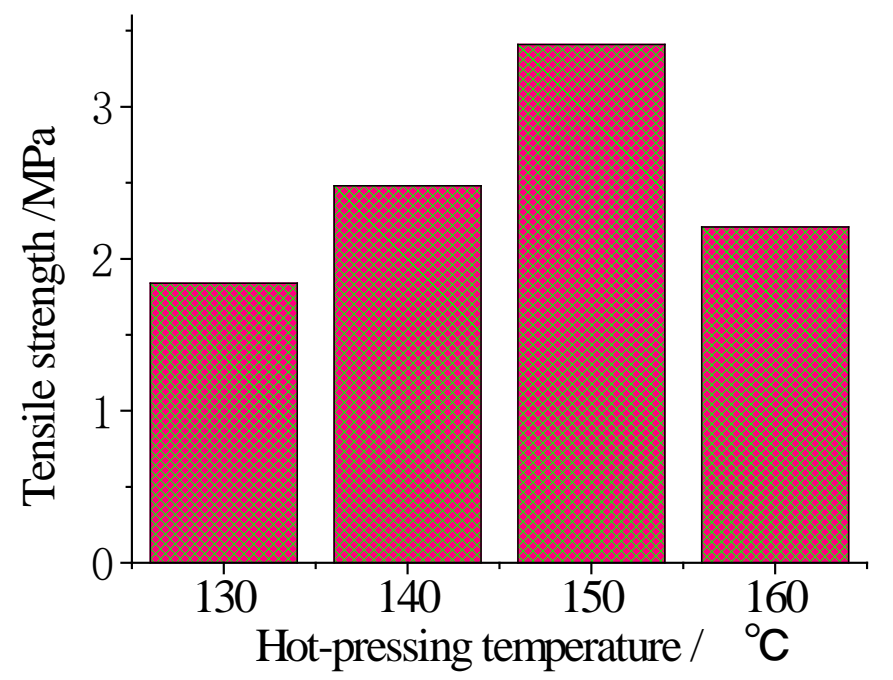

Fig. 4 The effect of hot-pressing temperature on tensile strength of composites.

\section{Summary}

Different parts of rice straw have different biological and mechanical characteristics. This indeed affects performance of composite. The best strength result was 3.67 MPa for PVA/LPRS composite, but in our previous study, best strength result was 5.29 MPa for composite prepared with upper part of rice straw.

\section{Acknowledgements}

This work was financially supported by Jiangxi Technology Supporting Project (20121BBE50008), Jiujiang Double Hundred and Double Thousand Talent Program, Jiujiang University Key Research Project (2014KJZD002).

\section{References}

[1] S.D. Zhu, W.J. Huang, W.X. Huang, et al: Appl. Energy Vol. 154(2015), p.190

[2] L. Zhang, Y.C. Hu: Mat. Design. Vol. 55(2014), P.19

[3] C.B. Wu, Y. Bo: Appl. Mechan. Mater. Vol. 525(2014), p.166

[4] N. Said, T. Bishara, A. García-Maraver and M. Zamorano: Waste Manage. Vol. 33(2013), p. 2250

[5] J.J. Liu, C.J. Jia and C.X. He: AASRI Procedia Vol. 3(2012), p. 83

[6] L.J. Qin, J.H. Qiu, M.Z. Liu, S.L. Ding, L. Shao, S.Y. Lü, G.H. Zhang, Y. Zhao, X. Fu: Chem. Eng. J. Vol. 166(2011), p. 772

[7] Y. Zhao, J.H. Qiu, H.X. Feng, M. Zhang, L. Lei, X.L. Wu: Chem. Eng. J. Vol. 173(2011), p. 659 